\title{
BMJ Open Mapping the evidence of intimate partner violence among women living with HIV/AIDS in Africa: a scoping review protocol
}

\author{
Mengistu Meskele, ${ }^{1}$ Nelisiwe Khuzwayo, ${ }^{2}$ Myra Taylor $^{3}$
}

To cite: Meskele M, Khuzwayo N, Taylor M. Mapping the evidence of intimate partner violence among women living with HIV/AIDS in Africa: a scoping review protocol. BMJ Open 2019;9:e029284. doi:10.1136/ bmjopen-2019-029284

- Prepublication history for this paper is available online. To view these files, please visit the journal online (http://dx.doi org/10.1136/bmjopen-2019029284).

Received 22 January 2019

Revised 04 July 2019

Accepted 10 July 2019
Check for updates

(C) Author(s) (or their employer(s)) 2019. Re-use permitted under CC BY-NC. No commercial re-use. See rights and permissions. Published by BMJ.

${ }^{1}$ School of Public Health, Wolaita Sodo University, Wolaita Sodo, Ethiopia

${ }^{2}$ School of Nursing and Public Health, Discipline of Rural Health, University of KwaZuluNatal, Durban, South Africa ${ }^{3}$ School of Nursing and Public Health, Discipline of Public Health, University of KwaZuluNatal, Durban, South Africa

Correspondence to Dr Mengistu Meskele; mengistumeskele@wsu.edu.et

\section{ABSTRACT}

Introduction Among women living with HIV, intimate partner violence (IPV) is increasingly recognised worldwide as a serious public health concern. The understanding of the link between IPV and HIV is currently inconclusive and information concerning the IPV experiences of HIV-infected women is insufficient. This protocol aims to map evidence of IPV against women living with HIV/AIDS in Africa. Methods and analysis We will search and review peerreviewed and review articles. The comprehensive search will include the electronic databases PubMed, MEDLINE with full text via EBSCO host, Google Scholar, Science Direct and Scopus. The advanced search will use MeSH terms. Grey literature will also be included. The titles of the studies from the database searches will be screened, and duplicates will be removed. The abstract screening will be done independently by two reviewers, followed by the fulltext screening which will be based on the eligibility criteria The six methodological stages in this review will be to: identify the research questions; identify relevant studies; select the studies; chart the data; collate, summarise and report the results; and thereafter undertake consultations. The quality of studies included in the review will be determined by the Mixed Methods Appraisal Tool. NVIV0 software V.11 will be used to undertake a thematic analysis of each of the studies and to extract the relevant outcomes.

Dissemination The results of this study will be disseminated through publication, and presented at conferences related to IPV.

Scoping review registration Currently, a scoping review is not eligible for registration on the International Prospective Register of Systematic Reviews.

\section{INTRODUCTION}

Women who are living with HIV and who disclose their HIV serostatus are more likely to experience intimate partner violence (IPV) than women who are HIV negative. ${ }^{1}$ Among the former IPV and HIV may provide overlapping or perhaps intersecting challenges. $^{2}$ The reason that women experience such violence is that the abusers have been shown to usually want to establish and maintain power and control over another person.

\section{Strengths and limitations of this study}

- The links between HIV/AIDS and intimate partner violence (IPV) are complex and not well understood; hence, this review aims to assess in-depth any associations among women living with HIV and the evidence obtained will provide a baseline for future research in gender-based violence.

- One of the limitations of this review will be that it only includes the literature published in the English language. Since the studies published in other languages will be missing, other evidence about IPV in women infected with HIV will be lacking.

- It is possible that our review will not include all articles which have been published in every journal since some may not be accessible.

This is often reflected in the imbalance of power between the women and their abusers. ${ }^{3}$ IPV is defined as '... any behaviour within an intimate relationship that causes physical, psychological or sexual harm to those in the relationship'. This also includes 'emotional abuse and controlling behaviour by an intimate partner'. ${ }^{4}$

Worldwide, IPV is of public health and social concern. The prevalence of IPV in the WHO's different regions varies, but is similar in the Eastern Mediterranean and South East Asian regions (where IPV was reported to be $37 \%$ and $37.7 \%$, respectively), to that in Sub-Saharan Africa, where the prevalence was $36.6 \% .^{5}$ Over a third of women in these regions reported IPV, and this high prevalence of IPV both among women and girls is of concern. ${ }^{25}$ The deleterious consequences of IPV among women and young girls are well documented, and this has been shown to affect their mental, sexual and reproductive health. ${ }^{5-7}$ Studies on IPV and depression show that women who are exposed to physical violence, childhood sexual abuse, mild or severe emotional violence, and where there is 
high spousal control are more likely to be depressed, ${ }^{6-8}$ more likely to abuse alcohol, ${ }^{5-7}$ give birth to babies of low weight. ${ }^{5-7}$ Since they may not be able to negotiate condom use ${ }^{5-7}$ they are also at risk of being infected with sexually transmitted infections (STIs) including HIV. ${ }^{5-79}$

The relationship of IPV and HIV among women and girls is a topic of intense debate within the scientific community. ${ }^{2} 10$ Some researchers consider that IPV increases women's vulnerability to HIV infection, ${ }^{1}{ }^{5-7}$ while other researchers suggest that HIV positive status among women may influence IPV. ${ }^{211}$ A study conducted in Zimbabwe among pregnant women living with HIV revealed an IPV prevalence of $40 \% .{ }^{12}$ Further, a study in Kenya showed that after post-HIV serostatus disclosure, one in three women experienced partner violence. ${ }^{13}$ Moreover, both the combination of physical and sexual IPV (OR: 2.00; 95\% CI: 1.24 to 3.22), but also experiencing any type of IPV were associated with HIV infection in women $(\mathrm{OR}=1.41 ; 95 \% \mathrm{CI}$ : 1.16 to 1.73$) .{ }^{14}$

IPV and HIV/AIDS are thus two pandemics that require integrated and collaborative interventions. The Joint United Nations Programme established new targets for the scale-up of HIV treatment by 2030, including increasing the number of people who know their HIV status by $90 \%$, increasing the number of people who receive sustained antiretroviral therapy by $90 \%$ and aiming to ensure that $90 \%$ of all the people who are HIV sero positive receive ART. ${ }^{15}$ These new targets are promising but achieving these goals could be compromised by women and young girls experiencing IPV. Therefore, this scoping review aims to map evidence of IPV among women living with HIV in Africa.

The information generated through this scoping review can be used by researchers, policy-makers and programme developers to develop appropriate programmes and policy frameworks.

\section{METHODS}

This review is part of a large-scale study on IPV among women living with and without HIV in Ethiopia. The review will use the framework which is proposed by Arksey and O'Malley ${ }^{16}$ which has six stages. These are: (1) identifying the research questions and defining the eligibility criteria, (2) conducting an extensive search and identifying relevant studies, (3) making the study selection and appraising the quality of the study (4) synthesising the included studies (charting the data) and presenting the findings by using a Preferred Reporting Items for Systematic Reviews and Meta-Analyses (PRISMA) chart, (5) collating, summarising and reporting and (6) consultation (box 1).

\section{Stage 1: identifying the research questions}

This scoping protocol is based on the following research questions:

1. Is there evidence of IPV experiences among women living with HIV in Africa?
Box 1 The proposed scoping review will follow the below steps

Stage 1: Formulating the study question.

Stage 2: Identifying the relevant studies.

Stage 3: Study selection.

Stage 4: Charting the data.

Stage 5: Collating, summarising and reporting.

Stage 6: Consultation.

2. Is there evidence that shows that HIV status disclosure influences IPV among women in Africa?

3. Is there evidence that socio-demographic characteristics are associated with IPV among women living with HIV in Africa?

\section{Eligibility criteria}

Inclusion criteria are:

- Studies with study participants aged 15 years and above.

- Studies with evidence of IPV against women.

- Studies with evidence about HIV positive persons.

- Year of publication: from 1 January 2009 to 1 April 2019. In order to obtain the maximum amount of updated information, studies on IPV over the past 10 years will be reviewed.

- Peer-reviewed literature, grey literature, government documents, policy briefs, systematic reviews and meta-analysis.

- Studies conducted in Sub-Saharan African countries (namely, all the countries in Africa except Algeria, Djibouti, Egypt, Libya, Morocco, Somalia, Sudan and Tunisia).

Exclusion criterion: Articles published in a language other than English.

The elements of the Population, Concept and Context criteria to map studies ${ }^{17}$ (table 1 ).

\section{Stage 2: identification of the relevant studies}

To search the literature, a Google form will be created to record the topic of the study, author and date, and a review questionnaire will be created. The Google form will enable screeners to record studies for screening that have been included in the endnote library.The keywords will be inserted into the PubMed advanced search menu and the results that appear will be selected and exported to Endnote. Furthermore, using a comprehensive search strategy, published evidences will be searched and included in this study. A variety of literature, including peer-reviewed articles and grey literature will be retrieved. The PubMed advanced search will use Mesh terms using terms such as IPV, women, HIV, Africa, domestic violence and date of publication after 1 January 2009 will be sought.

In addition to PubMed, MEDLINE with full text via EBSCO host, Google Scholar, Science Direct and Scopus will be used. As a result of the search to date, a total of 697 


\begin{tabular}{ll}
\hline Table 1 & PCC frame work \\
\hline PCC & Description \\
\hline P=Population & $\begin{array}{l}\text { Women living with HIV: The population for this study } \\
\text { will be all women aged } 15 \text { years and above, who are } \\
\text { either living with HIV and/or receiving antiretroviral } \\
\text { treatment. }\end{array}$ \\
$\begin{array}{l}\text { IPV (physical and/or sexual and/or emotional/ } \\
\text { psychological violence) or domestic violence. }\end{array}$ \\
$\begin{array}{l}\text { Sub-Saharan African countries, where most of the } \\
\text { problem of IPV exists among women living with HIV. }\end{array}$
\end{tabular}

IPV, intimate partner violence; PCC, Population, Concept and Context.

articles have been found from the PubMed search. The search was conducted on 8 April 2019 (table 2).

\section{Stage 3: study selection}

Screening

The search strategy will be piloted in order to check the appropriateness of the selected electronic database and the keywords we decided to include. The title screening will be conducted by the principal investigator and the abstract screening will be done independently by two reviewers. The purpose of the title screening is to assess the titles for eligibility and to remove duplicates. An Endnote library will be created and all the eligible titles will be exported to the library. Any duplicates which are identified will be removed before the screening of abstract. Relevant articles based on the inclusion criteria of the scoping review will be selected.

In order to minimise reporting bias, the abstract screening will be done independently by two reviewers. ${ }^{17}$ Moreover, the full-text article screening will be done independently, based on the eligibility criteria. Authors will be contacted for articles not available electronically. During the full article retrieval, the University of KwaZulu-Natal library will be used. When a disagreement arises among two reviewers, the third reviewer will make the decision. Update of the findings will be presented using a PRISMA chart (figure 1).

\section{Quality assurance of the study}

To determine the methodological quality, the Mixed Methods Appraisal Tool (MMAT) 2018 version will be adapted and used. Hence, each study will be evaluated using the MMAT tool. ${ }^{18}$ Moreover, the Preferred Reporting Items for Systematic Review and Meta-Analysis-Protocol
2015 and PRISMA extension for Scoping Reviews checklists will be used to check whether the recommended items will be used. ${ }^{19}{ }^{20}$ Moreover, during analysis, we will review the checklist of the COnsolidated criteria for REporting Qualitative research: a 32-item checklist for interviews and focus groups will be used. ${ }^{21}$

\section{Stage 4: charting the data}

Data charting table (table 3) will be used to extract the necessary background information and to extract information from each selected study. Data charting will be done using an Excel spreadsheet. The data charting form will highlight important ideas regarding the variables from the background. The research questions will be answered by the variables and the themes included in the form. We will continuously update data charting form during the duration of the study.

\section{Stage 5: collating, summarising and reporting the result}

Qualitative data analyses using thematic and NVIVO software V.11 will be used to collate, summarise and report the results. The researchers will read and reread the articles thoroughly noting down the initial ideas in order to find codes. Coding interesting features of the data in a systematic fashion across the entire articles and collating data relevant to each code will be done. We will develop the codes into potential themes. Finally, defining and naming of the themes and producing the report will be done. ${ }^{22}$ The description of the coding tree and thematic content analysis will be used to analyse the data. Data related to the IPV experience among HIV positive women will be extracted and coded. Emerging themes will be identified and the data will be coded according to the identified themes. The analysis process will use the following steps (1) Coding data from the selected articles, (2) categorising the codes into themes, (3) displaying the data, (4) identifying key patterns in the data and identifying sub-themes, (5) summarising and synthesising.

\section{Stage 6: consultation}

There will be consultation of the stakeholders, such as policy-makers, clinicians, patients and families, and any other appropriate group which has conducted research on IPV, in order to obtain more references and to

\section{Table 2 Results of pilot search in PubMed}

\section{Keywords searched}

$\begin{array}{lll}\begin{array}{l}\text { Date of } \\ \text { search }\end{array} & \begin{array}{l}\text { Search engine } \\ \text { used }\end{array} & \begin{array}{l}\text { Number of publication } \\ \text { retrieved }\end{array}\end{array}$

\footnotetext{
((((“intimate partner violence"(MeSH Terms) AND "women"(MeSH

Terms)) AND hiv(Title)) OR "domestic violence"(MeSH Terms)) AND

"africa”(MeSH Terms)) AND ("2009/01/01"(PDAT): “3000”(PDAT)

Intimate partner violence and its associated factors among women living with HIV/AIDS in Sub-Saharan Africa

Intimate partner, HIV, Sub-Saharan, Africa
}

8 April 2019 PubMed

697

Google scholar $\quad 7430$

EBSCO host

48 


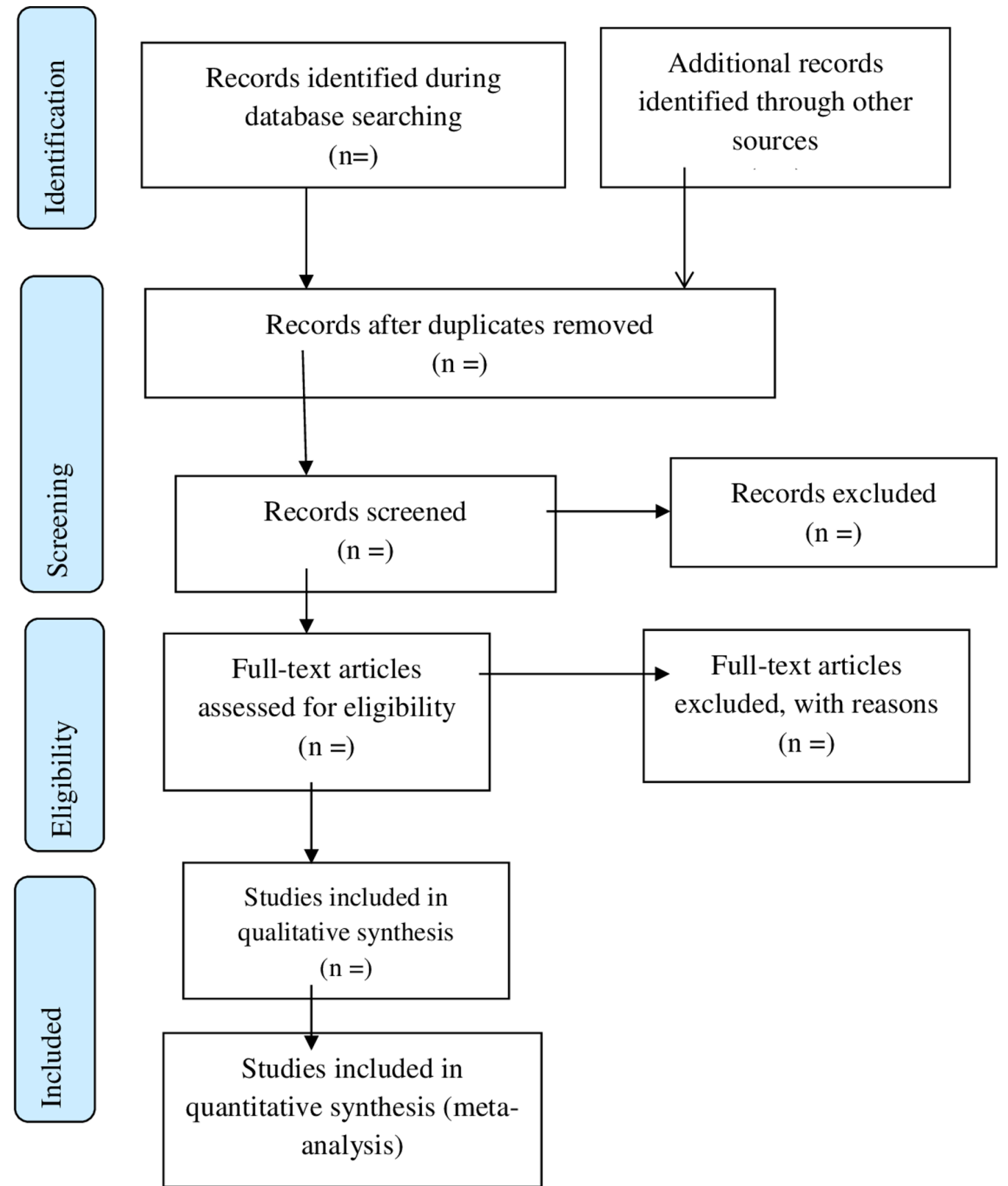

Figure 1 The Preferred Reporting Items for Systematic Reviews and Meta-Analyses 2009 flow diagram to update screening. Source: Moher et al. ${ }^{19}$

provide insights on what the literature fails to highlight (table 3$){ }^{23}$

\section{DISCUSSION}

Understanding evidence of the experience of IPV among women living with HIV in Africa is vital. The evidence from the current review may provide a framework that can improve the health of HIV positive women, develop advocacy strategies and strengthen women's rights. The links between HIV/AIDS and IPV against women are not completely understood. Moreover, IPV poses an indirect risk of HIV infection in different ways. IPV may increase sexual risk-taking and women with a history of violence may not be able to discuss condom use ${ }^{67}$ Fear of violence may prevent women from seeking HIV test, and this also prevents disclosure of their HIV status. Furthermore, women who experienced violence may delay in accessing to antiretroviral treatment (ART) for fear of further violence. ${ }^{67}$ Therefore, it is important to study violence against women who are living with HIV.

As revealed in the Sustainable Development Goals (SDG), gender discrimination is still a social norm in many nations. Achieving gender equality and empowering all women and girls were given due attention and emphasis 


\begin{tabular}{l} 
Table 3 Sample data extraction table \\
\hline Authors \\
\hline Year of publication \\
Country of origin \\
Aims of the study \\
Sample size \\
Methodology \\
Intervention \\
Outcomes measured \\
Duration of the intervention \\
Key findings which match the review \\
questions
\end{tabular}

by the SDGs. ${ }^{724}$ The current scoping review will map and document the existing evidence of IPV among HIV-positive women. The information from the review will help to understand the evidence of IPV among HIV-positive women in Africa. It will also map the evidence that HIV status contributes to IPV in Africa and the evidence as to whether disclosure of HIV-positive status leads to IPV. This review will focus on the studies published between 2009 and 2019. We chose these years because we want to obtain updated information; hence, we used the literature over the past 10 years, particularly focusing on the studies of women who are living with HIV. This is because their health needs have to be prioritised. The results from this scoping review will highlight key evidence of IPV against women who are living with HIV, and the evidence will also highlight the gaps and opportunities for future research. The result of the scoping review will also help to identify the priority areas for primary research on genderbased violence.

\section{Dissemination}

This study will be disseminated through publication in an open access peer-reviewed journal, printed and presented in international and national conferences related to IPV.

\section{Definition of terms}

IPV can occur among two people in a close relationship. The perpetrators include current and former spouses as well as dating partners. IPV includes four behaviours, namely physical violence, sexual violence, stalking and psychological aggression. ${ }^{25}$ Similarly, the WHO document on IPV includes physical and sexual violence, emotional abuse and controlling behaviours by partner. ${ }^{26}$ The definitions are detailed below.

\section{Intimate partner violence}

'Any act or omission by a current or former intimate partner which negatively effects the well-being, physical or psychological integrity, freedom, or right to full development of a woman. ${ }^{27}$

\section{Physical violence}

'The intentional use of physical force with the potential for causing death, injury, or harm. ${ }^{27}$

\section{Sexual violence}

'Any sexual act, attempt to obtain a sexual act, unwanted sexual comments or advances, or acts to traffic, or otherwise directed, against a person's sexuality using coercion, by any person regardless of their relationship to the victim, in any setting, including but not limited to home and work. ${ }^{28}$

\section{Psychological violence}

This 'occurs when someone says or does something to make a person feel stupid or worthless". 29

\section{Controlling behaviours by an intimate partner}

'Controlling behaviours, include acts to constrain a woman's mobility or her access to friends and relatives, extreme jealousy, etc' ${ }^{29}$

\section{Emotional abuse by an intimate partner}

Emotional abuse, or mental abuse (psychological abuse) 'is a form of abuse, characterized by a person subjecting or exposing another person to behaviour that may result in psychological trauma, including anxiety, chronic depression, or post-traumatic stress disorder'. ${ }^{30}$

\section{CONCLUSION}

The evidence of the link between IPV and HIV is currently inconclusive, and information regarding the IPV experience among HIV-infected women is inadequate. This review is part of a large-scale study on IPV among women living with and without HIV in Ethiopia. This study has a particular emphasis on IPV against women living with HIV in Africa. The review will use a framework which has six stages. It aims to assess whether there is evidence of IPV among HIV-positive women, whether HIV status contributes to IPV in Africa, and if the disclosure of HIV-positive status to one's partner leads to IPV in Africa. The result of the scoping review will also help to identify priority areas for primary research in gender-based violence.

\section{Patient and public involvement}

This study is a scoping review which was done without patient involvement. Patients were not invited to comment on the study design and were not consulted to develop patient-relevant outcomes or interpret the results. They were also not invited to contribute to the writing or editing of this document for readability or accuracy.

Acknowledgements We would like to acknowledge the UKZN Systematic Review Support Service at the University of KwaZulu-Natal, South Africa, for their support through the training workshop and assistance in protocol development for the review. Also, we would like to extend our gratitude to the School of Nursing and Public Health, College of Health Science, University of KwaZulu-Natal, for all their assistance and help. 
Contributors MM: conceived and drafted the study as part of his PhD study; NK and MT: supervised the protocol development and revisited the manuscript critically for important intellectual content. The three authors are involved in substantial contributions to conception and design. The three authors read and approved the final manuscript. MM, NK and MT are agreed to be accountable for all aspects of the work.

Funding The authors have not declared a specific grant for this research from any funding agency in the public, commercial or not-for-profit sectors.

Competing interests None declared.

Patient consent for publication Not required.

Provenance and peer review Not commissioned; externally peer reviewed.

Open access This is an open access article distributed in accordance with the Creative Commons Attribution Non Commercial (CC BY-NC 4.0) license, which permits others to distribute, remix, adapt, build upon this work non-commercially, and license their derivative works on different terms, provided the original work is properly cited, appropriate credit is given, any changes made indicated, and the use is non-commercial. See: http://creativecommons.org/licenses/by-nc/4.0/.

\section{REFERENCES}

1. Abdool Karim Q, Baxter C. The dual burden of gender-based violence and HIV in adolescent girls and young women in South Africa. S Afr Med J 2016;106:1151-3.

2. The White House Washington Working Group. Addressing the Intersection of HIV/AIDS, Violence against Women and Girls, \& Gender-Related Health Disparities.Washington, 2013. Available: www.hoccprograms.org/vaw-hiv_working_group_report_final_-_9-6-2013.pdf [Cited 13 Jul 2018].

3. Government of Newfoundland and Labrador. Violence prevention initiative: defining violence and abuse. Available: https://www.gov.nl. ca/VPI/types/ [Cited 13 Jul 2018].

4. WHO. Understanding and addressing violence against women: intimate partner violence, 2012. Available: https://apps.who.int/ iris/bitstream/handle/10665/77432/WHO_RHR_12.36_eng.pdf; jsessionid=4769C7FB9DE4C25AE8C0EFCCC68907DE? sequence $=1$ [Cited 04 Apr 2019].

5. WHO. Global and regional estimates of violence against women: prevalence and health effects of intimate partner violence and nonpartner sexual violence. World Health organization, 2013. Available: https://www.who.int/reproductivehealth/publications/violence/ 9789241564625/en/ [Cited 13 Jul 2018].

6. WHO. Violence against women and HIV/AIDS: critical Intersections intimate partner violence and HIV/AIDS. A UNAIDS initiative the global coalition on women and AIDS, 2009. Available: https://npin. cdc.gov/publication/intersection-intimate-partner-violence-and-hivwomen [Cited 13 Jul 2018].

7. CDC. Intersection of intimate partner violence and HIV in women, 2014. Available: https://npin.cdc.gov/publication/intersectionintimate-partner-violence-and-hiv-women [Cited 13 Jul 2018].

8. Deyessa N, Berhane Y, Alem A, et al. Intimate partner violence and depression among women in rural Ethiopia: a cross-sectional study. Clin Pract Epidemiol Ment Health 2009;5

9. El-Bassel N, Gilbert L, Krishnan S, et al. Partner violence and sexual HIV-risk behaviors among women in an inner-city emergency department. Violence Vict 1998;13:377-93.

10. Durevall D, Lindskog A. Intimate partner violence and HIV in ten sub-Saharan African countries: what do the demographic and health surveys tell us? Lancet Glob Health 2015;3:e34-43.

11. Gielen AC, McDonnell KA, Burke JG, et al. Women's lives after an HIV-positive diagnosis: disclosure and violence. Matern Child Health J 2000;4:111-20.
12. Shamu S, Zarowsky C, Shefer T, et al. Intimate partner violence after disclosure of HIV test results among pregnant women in Harare, Zimbabwe. PLoS One 2014;9:e109447.

13. Colombini $M$, James $C$, Ndwiga $C$, et al. The risks of partner violence following HIV status disclosure, and health service responses: narratives of women attending reproductive health services in Kenya. $J$ Int AIDS Soc 2016;19:20766.

14. Li Y, Marshall CM, Rees HC, et al. Intimate partner violence and HIV infection among women: a systematic review and meta-analysis. J Int AIDS Soc 2014;17:18845.

15. UNAIDS. Joint United nations programme on HIV/AIDS. 90-90-90 an ambitious treatment target to help end the AIDS epidemic. Available: https://www.unaids.org/sites/default/files/media_asset/90-90-90_en. pdf [Cited 28 Jun 2019].

16. Arksey H, O'Malley L. Scoping studies: towards a methodological framework. Int J Soc Res Methodol 2005;8:19-32.

17. Joanna B. Institute. Joanna Briggs Institute Reviewers' Manual: 2015 edition / Supplement. The Joanna Briggs Institute, 2015. Available: https://nursing.lsuhsc.edu/JBI/docs/ReviewersManuals/Scoping-.pdf [Accessed 12 Jan 2019].

18. Quan N, Pierre P, Sergi F, et al. Mixed methods appraisal tool (MMAT) version 2018. in family medicine mu, Canada, editor, 2018. Available: http://mixedmethodsappraisaltoolpublic.pbworks.com/w/file/fetch/ 127916259/MMAT_2018_criteria-manual_2018-08-01_ENG.pdf [Accessed 12 Jan 2019].

19. Moher D, Liberati A, Tetzlaff J, et al. Preferred reporting items for systematic reviews and meta-analyses: the PRISMA statement. PLoS Med 2009;6:e1000097.

20. Tricco AC, Lillie E, Zarin W, et al. PRISMA extension for scoping reviews (PRISMA-ScR): checklist and explanation. Ann Intern Med 2018;169:467-73.

21. Tong A, Sainsbury P, Craig J. Consolidated criteria for reporting qualitative research (COREQ): a 32-item checklist for interviews and focus groups. Int J Qual Health Care 2007;19:349-57.

22. Braun V, Clarke V. Using thematic analysis in psychology. Qual Res Psychol 2006;3:77-101.

23. Dijkers M. What is a scoping review? 2015. Available: http://ktdrr.org/ products/update/v4n1/ [Cited 13 Jul 2018].

24. UN. Resolution adopted by the general assembly on 25 September 2015.Transforming our world: the 2030 agenda for sustainable development, 2015. Available: http://www.un.org/ga/search/view_ doc.asp?symbol=A/RES/70/1\&Lang=E: [Cited 12 Jan 2019].

25. WHO. Understanding intimate partner violence: fact sheet, 2014. Available: https://www.healthymarriageandfamilies.org/libraryresource/understanding-intimate-partner-violence-fact-sheet-2014 [Cited 13 Jul 2018].

26. WHO. Intimate partner violence, 2012. Available: http://www.who.int/ reproductivehealth/publications/violence/rhr12_36/en/ [Cited 13 Jul 2018].

27. Mary Ellsberg LH. Researching Violence Against Women:A practical guide for researchers and activists. Washington DC, United States: World Health Organization, PATH, 2005. Available: http://apps. who.int/iris/bitstream/handle/10665/42966/9241546476_eng.pdf; jsessionid=470313F8B652FA010870002DEC07CF67? sequence $=1$ [Cited 13 Jul 2018].

28. WHO. Chapter 6. sexual violence, p. 149. Available: http://www.who. int/violence_injury_prevention/violence/global_campaign/en/chap6. pdf [Cited 13 Jul 2018].

29. WHO. WHO Multi-country Study on Women's Health and Domestic Violence against Women Initial results on prevalence, health outcomes and women's responses, 2005. Available: https://www. who.int/reproductivehealth/publications/violence/24159358X/en/ [Cited 13 Jul 2018].

30. Wikipedia: psychological abuse. Available: https://en.wikipedia.org/ wiki/Psychological_abuse\#cite_note-Emotional_Abuse-3 [Cited 13 Jul 2018]. 